\title{
Experimental Study on the Peroxidase Reacting Granules in the Blood Cells. The 11th Report of Histochemical Study of Peroxidase
}

\author{
By \\ Köichi Tsukamoto \\ Department of Anatomy, School of Dentistry, Nihon University, Tokyo. \\ (Director: Prof. T. Mitsui, M. D.)
}

\section{CONTENTS}

I. Introduction

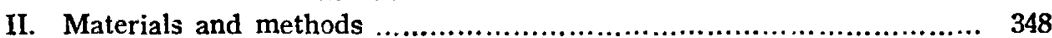

III. Results obtained ........................................................... 349

1. Influence of the light rays upon the peroxidase reaction ........... 349

2. Influence of the alcohol fixation upon the peroxidase reaction...... 352

3. Relation between the hydrogen peroxide solution and the peroxidase reaction

4. Relation between the $\mathrm{pH}$ value of the reagent used and the peroxidase reaction

5. Differences between the peroxidase reacting substances in the leukocytes and the erythrocytes................................... 356

6. The postmortem change of the peroxidase reaction of the blood cells both in man and animal ..................................... 358

7. The peroxidase staining of old blood smears ........................ 362

8. Water solubility of the peroxidase reacting granules ................. 363

9. The peroxidase reaction of the blood platelets ....................... 365

IV. Summary and conclusion ................................................ 365

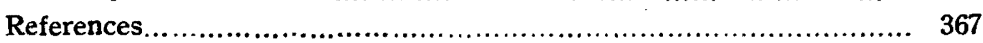

\section{Introduction}

The peroxidase reaction of the blood cells has been studied for years ${ }^{9}, 11,24,26,46$. In Japan, since 1922, Sato's School has published a large number of papers on the reaction using the copper method ${ }^{26}$ as well as A rakawa's method ${ }^{3}$ of milk peroxidase. At our laboratory, many histological studies have been made utilizing the NAS-Benzidine solution.

While experimenting with the inhibitors of peroxidase reacting granules in the blood cells, a strong inhibiting influence of the sunlight 
upon the reaction was identified, and the following new problems arose :

a) Differences in inhibition effect between visible and invisible rays.

b) Difference between ultraviolet and infra-red rays.

c) Difference between the peroxidase granules and May. Giemsa's special granules in the myeloid leukocytes.

d) The postmortem changes in the peroxidase reaction.

e) What are the peroxidase reacting granules in blood cells?

Of these, the last problem regarding the essential nature of the peroxidase reacting granules remains unanswered and requires further careful histological and biochemical studies. Therefore this investigation deals only with the histological findings of the blood cells under various conditions, and also may be regarded as one of steps toward a solution of the essential nature of the peroxidase or peroxidase reaction.

\section{Materials and Methods}

A great number of blood smears prepared from thirty human subjects, from four human cadavers, from five rabbits and from five guinea pigs, were used for this study. The blood smears were prepared from the circulating blood, heart blood, or bone marrow depending upon the purpose of the experiment. Blood smears up to three years of age were used to determine the stability of the peroxidase reacting granules in relation to drying. Specimens were taken from the human cadavers between twelve to twenty-nine hours after death. In these experiments, the NAS-Benzidine solution (NAS-B) was used for the peroxidase stain because of its simplicity and sensitivity. The latter solution is the mixture of $95 \mathrm{cc}$. of benzidine-hydrogen-peroxide solution with $5 \mathrm{cc}$. of one per cent aqueous solution of Nickel-AmmomiumSulfate (NAS) (techniques of the staining are described in the 1st Report of Histochemical Study of Peroxidase by Mitsui and Ikeda, 1951) ${ }^{13}$. The benzidine used here was prepared by E. Merck, Germany, and the hydrogen peroxide used is a three per cent aqueous solution. Four different filters were specially prepared for the experiments with the light rays, and the wave length of the light passing through them is described in detail in the following item. 


\section{Results obtained}

\section{Influence of the light rays upon the peroxidase reaction.}

It is widely known that the ultraviolet rays generally inhibit the function of the enzymes in various degrees, and that the oxidase reaction of leukocytes in the tissues is strongly influenced by the ultraviolet rays $^{8,20}$. However, the influence of the visible as well as invisible rays ${ }^{38}$ on the peroxidase staining of the blood cells has not adequately been studied. The effect of infra-red rays has not previously been reported. The purpose of this portion of the experiment was to determine whether the ultraviolet rays inhibit the peroxidase staining, and whether infra-red rays exert the same influence. In the experiments with the light rays, the human blood smears should not be fixed by alcohol or formalin, because fixatives generally exert an intense inhibiting influence upon the peroxidase staining. After exposure to the light rays, unfixed human blood smears were immediately stained with the NAS-Benzidine (NAS-B) solution.

The influence of the whole sunlight upon each blood cell is shown in table 1 , and that of various light rays is in table 2. These tables suggest that almost all light rays inhibit the peroxidase reaction of the leukocytes, and that the intensity of this inhibition depends on the wave length of the rays. The longer the wave length, the weaker becomes the inhibiting influence. It should be noted that these rays do not inhibit the peroxidase reaction of the erythrocytes, but rather activate it to some degree. This is one of important differences between the leukocytes and erythrocytes as will be described later. The detailed results of the experiments with the light rays are as follows:

Table 1. Effect of the sunlight upon the peroxidase reaction of human blood cells. $\mathrm{m} .=$ minutes, $\mathrm{h} .=$ hours. (NAS-Benzidine staining for 2 minutes)

\begin{tabular}{|l|c|c|c|c|c|c|c|c|c|c|c|}
\hline $\begin{array}{c}\text { acting } \\
\text { blood } \\
\text { cells }\end{array}$ & $0 \mathrm{~m}$. & $5 \mathrm{~m}$. & $10 \mathrm{~m}$. & $15 \mathrm{~m}$. & $30 \mathrm{~m}$. & $1 \mathrm{~h}$. & $\begin{array}{l}1 \mathrm{~h} . \\
30 \mathrm{~m} .\end{array}$ & $2 \mathrm{~h}:$ & $4 \mathrm{~h}$. & $6 \mathrm{~h}$. & $\begin{array}{c}7 \mathrm{~h} . \\
30 \mathrm{~m} .\end{array}$ \\
\hline neutrophils & + & + & + & + & + & \pm & - & - & - & - & - \\
\hline eosinophils & + & + & + & + & + & + & + & \pm & - & - & - \\
\hline erythrocytes & + & + & + & + & + & + & + & + & + & + & + \\
\hline
\end{tabular}


Table 2. Effect of various light rays upon the perxidase reaction of human blood cells. $\mathrm{m} .=$ minutes, $\mathrm{N}=$ neutrophils, $\mathrm{E}=$ eosinophils, $\AA={ }^{\circ}$ Angstrom. Intensity of the peroxidase reaction $(++=$ strong, $+=$ weak, $\pm=$ very weak, $-=$ negative $)$

\begin{tabular}{|c|c|c|c|c|c|c|c|c|c|c|}
\hline \multirow{3}{*}{ acting time } & \multicolumn{2}{|c|}{ sunlight } & \multicolumn{2}{|c|}{$\begin{array}{l}\text { pure ultra- } \\
\text { violet ray }\end{array}$} & \multicolumn{2}{|c|}{$\begin{array}{l}\text { with yellow } \\
\text { filter }\end{array}$} & \multicolumn{2}{|c|}{$\begin{array}{l}\text { with infra- } \\
\text { red filter }\end{array}$} & \multicolumn{2}{|c|}{$\begin{array}{l}\text { pure infra- } \\
\text { red ray }\end{array}$} \\
\hline & \multicolumn{2}{|c|}{$2700-23000 \AA$} & \multicolumn{2}{|c|}{$3150-3900 \AA$} & \multicolumn{2}{|c|}{ over $4500 \AA$} & \multicolumn{2}{|c|}{ over $7000 \AA$} & \multicolumn{2}{|c|}{$9500-13500 \AA$} \\
\hline & $\mathbf{N}$ & $\mathbf{E}$ & $\mathbf{N}$ & $\mathrm{E}$ & $\mathbf{N}$ & $\mathrm{E}$ & $\mathrm{N}$ & $\mathrm{E}$ & $\mathbf{N}$ & $\mathbf{E}$ \\
\hline no exposure & ++ & ++ & $t+$ & $+t$ & $+t$ & ++ & ++ & $+t$ & ++ & ++ \\
\hline $30 \mathrm{~m}$. & + & ++ & + & $+t$ & $+t$ & ++ & $+t$ & ++ & $+t$ & ++ \\
\hline $60 \mathrm{~m}$. & \pm & + & \pm & + & + & + & + & + & + & $+t$ \\
\hline $90 \mathrm{~m}$. & - & + & \pm & \pm & + & \pm & + & + & + & $+t$ \\
\hline $120 \mathrm{~m}$. & - & \pm & - & \pm & \pm & \pm & + & \pm & + & ++ \\
\hline
\end{tabular}

1) Sunlight. The experiment with the sunlight was carried out in May and in August. The wave lengths contained in the sunlight reaching the earth range from $2,700 \AA$ to $23,000 \AA$. . Other wave lengths in the sunlight are absorbed while passing through the air. The whole sunlight, as shown in the tables, exerted the greatest influence upon the peroxidase staining of the leukocytes, in which case, however, the erythrocytes were entirely stable and showed fine blue peroxidase positive granules. Furthermore it is of prominent interest that neutrophils, monocytes, eosinophils and basophils stained very well with Wright's or May-Giemsa's stain, although their 'peroxidase reactions were inhibited by sunlight.

2) The influence.of the sunlight through the common glass. The wave lengths contained in this filtered light ray lie between $2,700 \AA$ and $20,000 \AA$. The result of the experiment with this filtered sunlight showed a similar but somewhat weaker influence upon the peroxidase reaction as compared with the whole sunlight.

3) Ultraviolet rays. These are invisible rays of the spectrum outside the violet end of the visible spectrum, and are also called chemical rays. The rays of $3150-3900 \AA$ range used were obtained from the sunlight by means of a special filter. These ultraviolet rays exerted greater influence upon the peroxidase staining capacity of the blood cells than either visible rays or infra-red rays. In this case, the neutrophil leukocytes become entirely peroxidase negative, while the eosinophils are slightly more stable. Their large coarse granules stain 
faintly brown with the NAS-B solution. On the contrary, the peroxidase reaction of the erythrocytes is not inhibited by ultraviolet rays, rather numerous blue granules are clearly seen within the cytoplasm, and these erythrocytes show a stronger peroxidase reaction than controls. The latter reaction is probably due to a chemical change of the hemoglobin caused by the light rays. This stability of the peroxidase reaction of the erythrocytes was confirmed in all the experiments with the sunlight, ultraviolet, visible, and infra-red rays.

4) Visible rays. In this experiment, two different filters were used in order to remove the ultraviolet rays from the sunlight. One is yellow filter (Luminous filter, Riken No. 102, Tokyo, or $Y_{2}$ filter, Riken Lens Industrial Co., Tokyo), another is infra-red filter (Ultrasin filter, Riken No. 9, Tokyo). The wave lengths of the visible rays lie between ca. $4,000 \AA$ and $7,600 \AA$, and through this yellow filter $\left(\mathrm{Y}_{2}\right)$ pass the rays of $4,500-\mathrm{ca} .20,000 \AA$, while the rays of $7,000-\mathrm{ca}$. $20,000 \AA$ pass through the infra-red filter (No. 9 mentioned above). Therefore, strictly speaking, the rays used here are the mixture of both the visible and infra-red rays. The influence of these rays is shown in table 2. As compared with the ultraviolet rays, the visible rays are less inhibitive to the peroxidase reaction of the blood cells. The visible rays possess an inhibiting effect upon the peroxidase reaction, as shown by the more intense influence of a combination of visible and infra-red rays than that obtained by pure infra-red rays, as demonstrated in table 2.

5) Infra-red rays. This is the part of the invisible spectrum contiguous to the red end of the visible spectrum, comprising radiations of greater wave length than that of red light. The infra-red rays are also called heat rays. The infra-red rays, whose wave lengths are over ca.' $7,600 \AA$, were obtained from the sunlight by means of the specially-made infra-red filter $(\mathrm{S})$. The rays containing the wave lengths between $9,500 \AA$ and $13,500 \AA$ (maximum 11,600 $\AA$ ), passed through this filter $(\mathrm{S})$, ultraviolet and visible rays were completely removed. This experiment demonstrated that the infra-red rays exert the least influence upon the peroxidase reaction of the blood cells. Furthermore, even this slight influence of the infra-red rays, was probably due to the heating energy of the ray, because the blood cells became peroxidase negative only when the blood smear preparations were heated over $45^{\circ} \mathrm{C}$ in the experiment with the infra-red rays. On the contrary, the peroxidase reaction of the blood cells were always 
inhibited by ultraviolet rays to a great degree even in cases where the blood smear preparation were not heated above $37^{\circ} \mathrm{C}$.

The peroxidase reacting granules in the leukocytes generally stain blue with the NAS-B solution. However, with greater exposure to the rays, this blue color of the granules in the neutrophils changes into dark violet, black, and into the so-called double phase (see the 2nd Report) ${ }^{14.18}$, next brown, finally the granules become fewer in number. until eventually all have disappeared. On the other hand, the blue granules in the eosinophils change to black, the double phase, brown, yellow or green, finally into peroxidase negative. The crystals of the benzidine derivatives appear very often within as well as around the leukocytes in the degenerating stages. Granules possessing a peroxidase affinity decrease in number and gradually disappear in leukocytes following these exposures. The same granules in erythocytes increase in number after exposure. These alterations are apparent even though the Wright's staining remain uniform through the experiment. In short, as to the intensity of these inhibiting influence of the light rays, the following order may be given: Ultraviolet, visible and infra-red rays. The ultraviolet rays are those of the shortest wave length and of the greatest inhibiting power of the peroxidase reaction in the leukocytes. Again, as to the influence of the heat ray, detailed results have already been reported by the author in the fifth report of histochemical study of peroxidase (1952). ${ }^{15,40.41}$

\section{Influence of the alcohol fixation upon the peroxidase reaction.}

Proper and immediate fixation is the most important step in the cytological studies. Formalin, alcohol, acetone or Zenker's solution are generally employed in routine work. After fixing blood smears in methanol and ethanol for 10 minute, 6 hour and 25 hour periods, granules possessing NAS-Benzidine affinity could be constantly demonstrated in erythrocytes but poorly in leukocytes. Methanol in all time periods completely inhibits the reaction in granulocytes. The inhibition effect of ethanol is proportional to the length of exposure and is complete in 25 hours in the neutrophil leukocyte (table 3). However, when fixing the blood smears with methanol for one to two minutes, the peroxidase reaction of the leukocytes still occurs. The eosinophils are slightly more stable to the alcohol fixation than neutrophils. As to the strong peroxidase reaction of the erythrocytes, further studies will be required, however, the chemical change may arise from hemo- 
Tab'e 3. Effect of the alcohol fixation upon the peroxidase staining of human blood cells (The staining was done with NAS-B solution for two minutes, $\min .=$ minutes, $h_{.}=$hours)

\begin{tabular}{|c|c|c|c|c|c|c|}
\hline \multirow{2}{*}{ fixatives } & \multirow{2}{*}{$\begin{array}{l}\text { fixing } \\
\text { time }\end{array}$} & \multicolumn{3}{|c|}{ peroxidase staining } & \multicolumn{2}{|c|}{ Wright's staining } \\
\hline & & $\begin{array}{l}\text { neutrophil } \\
\text { granules }\end{array}$ & $\begin{array}{l}\text { eosinophil } \\
\text { granules }\end{array}$ & $\begin{array}{l}\text { erythro- } \\
\text { cytes }\end{array}$ & $\begin{array}{l}\text { neutrophil } \\
\text { granules }\end{array}$ & $\begin{array}{l}\text { eosinophil } \\
\text { granules }\end{array}$ \\
\hline \multirow{3}{*}{ methanol } & $10 \mathrm{~min}$. & - & - & ++ & + & + \\
\hline & $6 \mathrm{~h}$. & - & - & ++ & + & + \\
\hline & $25 \mathrm{~h}$. & - & - & ++ & + & + \\
\hline \multirow{3}{*}{ ethanol } & $10 \mathrm{~min}$. & + & + & ++ & + & + \\
\hline & $6 \mathrm{~h}$. & \pm & \pm & $+t$ & + & + \\
\hline & $25 \mathrm{~h}$. & - & \pm & ++ & + & + \\
\hline
\end{tabular}

globin alterations during alcohol fixation. As previously reported by Sasaki $(1952)^{25}$, methanol shows the strongest inhibiting influence upon the peroxidase reaction of the leukocytes when compared with fixatives such as ethanol, acetone and formalin ${ }^{31}$.

\section{Relation between the hydrogen peroxide solution and the peroxidase reaction.}

The hydrogen peroxide $\left(\mathrm{H}_{2} \mathrm{O}_{2}\right)$ solution is the most important component in the peroxidase staining reagent. The peroxidase granules, according to the theory heretofore proposed, catalyze the reduction of hyrogen peroxide releasing an oxygen molecule which in turn oxidizes the hydrogen donor (e.g. benzidine). The peroxidase staining capacity of the blood cells changes in varying degrees dependent upon the amount of $\mathrm{H}_{2} \mathrm{O}_{2}$ present in the peroxidase stain. The results of the experiments are shown in table 4 , in which case the peroxidase staining was carried out with the mixture of both benzidine and $\mathrm{H}_{2} \mathrm{O}_{2}$ as in Graham's method, and the $\mathrm{H}_{2} \mathrm{O}_{2}$ solution used is ca. three per cent aqueous solution of hydrogen peroxide. The results in table 4 show that too much $\mathrm{H}_{2} \mathrm{O}_{2}$ solution in the reagent inhibits the peroxidase reaction of all blood cells as in the peroxidase reaction by means of the biochemical method ${ }^{10}$. The following results were also demonstrated by the experiment: 1) When the $\mathrm{H}_{2} \mathrm{O}_{2}$ is relatively small in amount, only the leukocytes become peroxidase positive while the erythrocytes 
remain negative. 2) In all instances the peroxidase reaction of the eosinophils is more intense than that of the neutrophils, in other words, the eosinophils is more stable to a large amount of $\mathrm{H}_{2} \mathrm{O}_{2}$ than the neutrophils. 3) When the benzidine solution is mixed with an equal amount of $\mathrm{H}_{2} \mathrm{O}_{2}$, the erythrocytes generally become peroxidase positive, while all leukocytes become negative. It was also confirmed by another experiment that the excessive $\mathrm{H}_{2} \mathrm{O}_{2}$ can inhibit the reaction only when the $\mathrm{H}_{2} \mathrm{O}_{2}$ is mixed with the benzidine solution, in other words, the $\mathrm{H}_{2} \mathrm{O}_{2}$ solution alone cannot inhibit the peroxidase reaction of blood cells. However, it is not clearly determined whether the decrease of the peroxidase reaction above mentioned was due to the formation of peroxidase $-\mathrm{H}_{2} \mathrm{O}_{2}$ compound or to the destruction of the peroxidase reacting granules.

Table 4. Effect of incressing concentrations of hydrogen peroxide upon the peroxidase staining of human blood cells. (Staining time is one minute).

+ (peroxidase positive), - (peroxidase negative reaction).

\begin{tabular}{|c|c|c|c|c|c|}
\hline & \multicolumn{2}{|c|}{ peroxidase stains } & \multirow[b]{2}{*}{ neutrophils } & \multirow[b]{2}{*}{ eosinophils } & \multirow[b]{2}{*}{ erythrocytes } \\
\hline & $\begin{array}{l}0.5 \% \text { aqueous } \\
\text { solution of } \\
\text { benzidine }\end{array}$ & $\begin{array}{c}\text { hydrogen } \\
\text { peroxide } \\
(3 \%)\end{array}$ & & & \\
\hline A & \multicolumn{2}{|c|}{$10 \mathrm{cc} .+1 \mathrm{gt}$} & $\cdot+$ & + & - \\
\hline B & \multicolumn{2}{|c|}{$9.5 \mathrm{cc} .+0.5 \mathrm{cc}$} & \pm & + & - \\
\hline C & \multicolumn{2}{|c|}{$9.0 \mathrm{cc} .+1.0 \mathrm{cc}$. } & \pm & + & - \\
\hline $\mathbf{D}$ & \multicolumn{2}{|c|}{$8.0 \mathrm{cc} .+2.0 \mathrm{cc}}$. & - & \pm & - \\
\hline $\mathbf{E}$ & \multicolumn{2}{|c|}{$7.0 \mathrm{cc} .+3.0 \mathrm{cc}}$. & - & - & \pm \\
\hline $\mathrm{F}$ & \multicolumn{2}{|c|}{$.6 .0 \mathrm{cc} .+4.0 \mathrm{cc}}$. & - & - & + \\
\hline G & \multicolumn{2}{|c|}{5.0 cc. +5.0 cc. } & - & - & + \\
\hline $\mathrm{H}$ & \multicolumn{2}{|c|}{4.0 cc. +6.0 cc. } & - & - & \pm \\
\hline I & \multicolumn{2}{|c|}{$3.0 \mathrm{cc} .+7.0 \mathrm{cc}}$. & - & - & - \\
\hline $\mathrm{J}$ & \multicolumn{2}{|c|}{2.0 cc. +8.0 cc. } & - & \multirow{2}{*}{-} & - \\
\hline $\mathrm{K}$ & \multicolumn{2}{|c|}{$1.0 \mathrm{cc} .+9.0 \mathrm{cc}}$. & - & & - \\
\hline
\end{tabular}

4. Relation between the $\mathrm{pH}$ value of the reagent used and peroxidase reaction.

The chemical reactions occur at a definite rate depending on the temperature, concentration and $\mathrm{pH}$ value of reactant etc. 
The peroxidase staining of blood cells mentioned above is one of histochemical reactions, therefore there is no doubt that the $\mathrm{pH}$ value of the reagent, such as benzidine $-\mathrm{H}_{2} \mathrm{O}_{2}$ solution or various salts, exerts much influence upon the peroxidase staining ${ }^{23.44}$. Of the many peroxidase stains recommended, each has an optimal $\mathrm{pH}$ value for best results, for example 4.4 in copper sulfate method, 5.4 in zinc sulfate method, 5.6-5.8 in NAS-B solution. Accordingly it can be said that the $\mathrm{pH}$ value of the peroxidase stain usually ranges from 4.0 to 6.0 , and that the peroxidase staining used in routine work corresponds to the acid peroxidase reaction (Mitsui). But that the peroxidase reaction of the blood cells can occur in an alkaline reagent ${ }^{30}$, is shown in the following experiment.

The wide variation in the $\mathrm{pH}$ values of the benzidine $-\mathrm{H}_{2} \mathrm{O}_{2}$ solution $(100 \mathrm{cc} .+10 \mathrm{gtt})$ was obtained by the use of 10 per cent citric acid, acetic acid or caustic potash solution. But of these, the solution having $\mathrm{pH}$ of 5.6 in table 5 , is NAS.B solution consisting of benzidine,

Table 5. Relation between the $\mathrm{pH}$ value of benzidine $-\mathrm{H}_{2} \mathrm{O}_{2}$ solution and the peroxidase staining of human blood cells (acidified with 10 per cent citric acid or acetic acid, alkalified with 10 per cent caustic potash).

\begin{tabular}{|c|c|c|c|c|c|}
\hline $\mathrm{pH}$ & lymphocyte & monocyte & neutrophils & eosinophils & erythrocytes \\
\hline 4.6 & - & - & - & \pm & + \\
\hline 5.6 & - & + & + & + & + \\
\hline 6.6 & - & + & + & + & - \\
\hline 8.0 & - & + & + & + & - \\
\hline
\end{tabular}

$\mathrm{H}_{2} \mathrm{O}_{2}$ and nickel-ammonium sulfate (NAS). Before staining with these solutions, the blood smears are fixed by ethanol for one minute to prevent cellular destruction when exposed to the benzidine- $\mathrm{H}_{2} \mathrm{O}_{2}$ solution, especially under alkaline conditions. The results obtained are summarized in table 5 . It is interesting to note that the peroxidase reaction depends not only on the $\mathrm{pH}$ value, but also on the different cations or anions used for the adjustment of the $\mathrm{pH}$ value-this is clear from the fact that the optimal $\mathrm{pH}$ value of the peroxidase reaction is not always in accord with each other depending on the buffers used. It was made clear by table 5 that the erythrocytes are peroxidase negative under neutral or alkaline conditions, while the myeloid leukocytes are positive under both acid and alkaline conditions. The ery- 
throcytes are positive only in the acid peroxidase reaction producing acid hematin, while the leukocytes are positive both in the acid and alkaline peroxidase reaction (Mitsui). At the $\mathrm{pH}$ of 4.6, erythrocytes are positive, while lymphocytes, monocytes and neutrophils are all negative; only eosinophils are weakly positive as shown in table 5 . This may represent one of the evidences of greater stability of eosin. ophil granules.

The peroxidase granules stained with the reagent containing benzidine are generally blue or brown. The granules stain brown under neutral, weakly acid or alkaline conditions. On staining with the acid peroxidase stain, the peroxidase reacting granules in the myeloid leukocytes stain brown at first, next show the so.called double phase (mixture of blue and brown), and finally change blue with increases of staining time. Both blue and brown granules can simultaneously occur at $\mathrm{pH} 5.6$ as already reported by Mitsui and Ikeda (see the 2nd Report of Histochemical Study of Peroxidase) ${ }^{14}$.

It is inferred that these findings are due to the chemical changes of the benzidine. Yellow, brown, green, blue, purple, black and other transitional colors develop according to conditions such as the $\mathrm{pH}$ value of the solution and the grade of the oxidation. On the other hand, it was already found by Bansi and Ucko (1927) 4 that there was no definite $\mathrm{pH}$ for the reaction of the peroxidase, but that the optimum $\mathrm{pH}$ would differ according to the materials used, so that for the same peroxidase the optimum $\mathrm{pH}$ was $4.5-6.5$ in the case of guaiacol, 3.5-5.0 in the case of cresol, and 6.5-7.0 in the case of pyrogallol. Hence, the optimum $\mathrm{pH}$ for the peroxidase varies according to the hydrogen donors used ${ }^{2}$.

\section{Differences between the peroxidase reacting substances in the leukocytes and erythrocytes.}

The peroxidase reaction of the leukocytes is similar to the erythrocytes.' Essentially both the peroxidase and hemoglobin belong to the hemoproteins, and the purpurogallin number (P.N.) of hemoglobin has been calculated as in case of the peroxidase ${ }^{12,21}$. The peroxidase of the leukocytes (myeloperoxidase or verdoperoxidase) was isolated by $\mathrm{Agner}$, K. $(1941)^{1.2}$ in the amorphous state, obtaining the P.N. value of 75 . The similarity between these two is also found in hematological study to some extent. For instance, the peroxidase reacting fine blue granules appear within the erythrocytes when the staining time is prolonged than in leukocytes using the Sato-Sekiy a's (copper) ${ }^{26.27}$, Usa mi-Yama- 
Experimental Study on the Peroxidase Reacting Granules in the Blood Cells.

moto's (zinc) ${ }^{45}$ or Mitsui-Ikeda's (NAS) ${ }^{13}$ staining methods. Again, the reagents for hemoglobin and for peroxidase in leukocyte are essentially the same but they differ only in the amount of $\mathrm{H}_{2} \mathrm{O}_{2}$ contained in each. However, the peroxidase reacting substance of erythrocytes distinctly differs from that of leukocytes in the stabilities to various inhibitors as shown in table 6 . This table was obtained by

Table 6. Peroxidase reaction of human blood cells under various conditions.

\begin{tabular}{|l|l|l|l|}
\hline whole sunlight & neutrophils & eosinophils & erythrocytes \\
\hline ultraviolet rays (3150-3900 A) & inhibiting & inhibiting & stable \\
\hline visible and infra-red rays & inhibiting & stable \\
\hline infra-red ray (9500-13500 A) & inhibiting & inhibiting & stable \\
\hline heating in incubator & inhibing in a & stable & stable \\
\hline reagent containing much $\mathrm{H}_{2} \mathrm{O}_{2}$ & inhibiting & inhibiting & stable \\
\hline reagent containing little $\mathrm{H}_{2} \mathrm{O}_{2}$ & react rapidly & react rapidly & react slowly \\
\hline methanol & inhibiting & inhibiting & stable \\
\hline ethanol & inhibiting & inhibiting & stable \\
\hline alkaline peroxidase reaction & positive & positive & negative \\
\hline acid peroxidase reaction & positive & positive & positive \\
\hline
\end{tabular}

summarizing the results already described, and by this, the following three points became obvious: 1) The erythrocytes possess more stable peroxidative activity than the leukocytes, namely, the peroxidase reacting substance of the erythrocytes is light rays-, heat-, $\mathrm{H}_{2} \mathrm{O}_{2^{-}}$, alcoholstable. 2) Generally speaking, the leukocytes possess more labile peroxidative activities than erythrocytes except in an alkaline environment. 3) The peroxidase reacting substance of the eosinophils is slightly more stable to the inhibitors mentioned above than that of the neutrophils.

These differences between the erythrocytes and leukocytes are probably due to their proteins corresponding to the "Apoenzyme" in enzymology. It is of prominent interest that the granules in the leukocytes, which became entirely peroxidase negative after exposure 
to inhibitors such as ultraviolet rays or alcohol fixation, still stained very finely with the May-Giemsa's stain ${ }^{16.17}$.

\section{The postmortem change of the peroxidase reaction of the blood cells both in man and animal.}

The fate of the neutrophils in the circulating blood which have fulfilled their purpose is not definitely known. Those about to die differ somewhat from the rest and are called senile. It is possible that they are more fragile, just as old people are. Sabin $(1923)^{7}$ noted that such presumably dying leukocytes are further characterized by partial loss of their affinity for vital dyes. Most of the dead cells are probably either autolysed in the blood stream or filtered out in the spleen and liver. Some may pass through the mucous membrane

Table 7. Short-timed peroxidase reaction of blood leukocytes in corpses (after M. Chiba)

\begin{tabular}{|c|c|c|c|c|}
\hline No. & $\begin{array}{c}\text { Age } \\
\mathrm{m} .=\text { months } \\
\mathrm{y} .=\text { years }\end{array}$ & Diagnosis & $\begin{array}{l}\text { Peroxidase } \\
\text { staining time } \\
\text { required } \\
\text { (seconds) }\end{array}$ & $\begin{array}{c}\text { Approximate } \\
\text { numbers of } \\
\text { hours after } \\
\text { death }\end{array}$ \\
\hline 1 & $6 \mathrm{~m}$. & Pneumonia + pyothorax & 9 & 10 \\
\hline 2 & $1 \mathrm{~m}$. & Pneumonia & 3 & 2 \\
\hline 3 & 10 y. $5 \mathrm{~m}$. & Lymphatic aleukemia & 3 & 14 \\
\hline 4 & $8 \mathrm{y}$. & Diphtheria & 3 & $3 \cdot$ \\
\hline 5 & $4 \mathrm{~m}$. & $\begin{array}{l}\text { Congenital syphilis } \\
\quad+\text { bronchopneumonia }\end{array}$ & 3 & 6 \\
\hline 6 & 1 y. 9 m. & Myeloid aleukemia & 3 & 12 \\
\hline 7 & $2 \mathrm{y}$. & Pyothorax & 3 & 9 \\
\hline 8 & $15 \mathrm{y}$. & Miliary tuberculosis & 5 & 15 \\
\hline 9 & $33 \mathrm{y.}$ & Syphilis + hysterism & 3 & 10 \\
\hline 10 & $28 \mathrm{y}$. & Pulmonary tuberculosis & 3 & 15 \\
\hline 11 & $21 \mathrm{y}$. & Puerperal feber & 3 & 12 \\
\hline 12 & 17 y. & Meningitis tuberculosa & 3 & 12 \\
\hline 13 & $17 \mathrm{y}$. & Myeloid leukemia & 3 & 12 \\
\hline 14 & $23 \mathrm{y}$. & $\begin{array}{l}\text { Pulmonary, abdominal and } \\
\text { laryngeal tuberculosis }\end{array}$ & 3 & 7 \\
\hline
\end{tabular}


Experimental Study on the Peroxidise Reacting Granules in the Blood Cells.

although the wandering leukocytes on the various mucosae are not always senile and effete. It is thought that the span of life of the lymphocyte may be less than twenty-four hours while that of the myeloid leukocytes may be a little greater. Neutrophils whose peroxidase reactions are very weak or entirely negative are occasionally found in the circulating blood. However, all these findings are descriptions of the leukocytes in the living body. In this portion of the paper, postmortem changes of the human and animal leukocytes were examined with special reference to their peroxidase reaction ${ }^{33}$. Sato, S $(1951)^{29}$ reported that the postmortem degenerative changes of the granules in pseudoeosinophils of guinea pigs occurred within twentyfour hours after death. On the other hand, Chiba $(1932)^{5,6}$ examined the postmortem changes of blood cells in human corpses utilizing Sato's peroxidase staining method, and stated that most of the blood cells in various corpses did not show any prolongation of the normal peroxidase staining time as shown in table 7 , namely no remarkable change of the reaction was found at least within fifteen hours after death. The results obtained in this experiment of postmortem changes are shown in three tables; the alterations of the leukocytes in the

Table 8. The postmortem change of the peroxidase staining of rabbits' leukocytes. $\mathrm{NB}=$ peroxidase staining with NAS-Benzidine solution, $\mathrm{MG}=$ May-Giemsa's staining, numbers of $(t)$ mean the intensity of the staining, $h .=$ hour.

Staining time of NB is 2 minutes.

\begin{tabular}{|c|c|c|c|c|c|c|c|c|c|c|}
\hline No. & $\begin{array}{l}\text { weight } \\
\text { of body } \\
\text { (gram) }\end{array}$ & $\begin{array}{l}\text { stain- } \\
\text { ing }\end{array}$ & $\begin{array}{l}\text { before } \\
\text { death }\end{array}$ & $\begin{array}{c}\text { immediately } \\
\text { after } \\
\text { death }\end{array}$ & $\begin{array}{l}3 \mathrm{~h} . \\
\text { after } \\
\text { death }\end{array}$ & $6 \mathrm{~h}$. & $12 \mathrm{~h}$. & $24 \mathrm{~h}$. & $36 \mathrm{~h}$. & $48 \mathrm{~h}$. \\
\hline \multirow{2}{*}{1} & \multirow{2}{*}{3260} & NB & +++ & +++ & +++ & + & + & + & \pm & - \\
\hline & & MG & +++ & +++ & $++t$ & ++ & ++ & + & + & - \\
\hline \multirow{2}{*}{2} & \multirow{2}{*}{3006} & NB & +++ & +++ & ++ & + & + & \pm & + & - \\
\hline & & MG & +++ & $++t$ & ++ & + & + & + & - & - \\
\hline \multirow{2}{*}{3} & \multirow{2}{*}{2714} & $\mathrm{NB}$ & ++ & ++ & + & + & + & + & - & 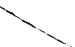 \\
\hline & & MG & +++ & +++ & ++ & + & + & \pm & - & $y$ \\
\hline \multirow{2}{*}{4} & \multirow{2}{*}{2270} & $\mathrm{NB}$ & +++ & +++ & + & + & + & \pm & - & 入 \\
\hline & & MG & ++ & ++ & ++ & + & + & + & + & \\
\hline \multirow{2}{*}{5} & \multirow{2}{*}{1850} & NB & ++ & & & & & & $\checkmark$ & \pm \\
\hline & & MG & ++ & & & & & & & + \\
\hline
\end{tabular}


rabbit's heart blood are shown in table 8 , those in the guinea pig's heart blood are in table 9, and those in the human heart blood and conjunctiva are in table 10 , respectively. In summarizing these results, the following points can be made:

Table 9. The postmortem change of the peroxidase staining of guinea pigs' leukocytes.

NB = peroxidase staining with NAS-Benzidine solution, MG=May-Giemsa's staining, numbers of $(t)$ mean the intensity of the staining, $h_{\text {. }}=$ hour.

Staining time of NB is 2 minutes.

\begin{tabular}{|c|c|c|c|c|c|c|c|c|c|c|c|}
\hline No. & $\begin{array}{l}\text { weight } \\
\text { of body } \\
\text { (gram) }\end{array}$ & $\begin{array}{c}\text { stain- } \\
\text { ing }\end{array}$ & $\begin{array}{l}\text { before } \\
\text { death }\end{array}$ & $\begin{array}{l}\text { immediately } \\
\text { after } \\
\text { death }\end{array}$ & $\mid \begin{array}{ll}3 \mathrm{~h} . \\
\text { after } \\
\text { death }\end{array}$ & $6 \mathrm{~h}$. & $12 \mathrm{~h}$. & $24 \mathrm{~h}$. & $36 \mathrm{~h}$. & $48 \mathrm{~h}$. & 60 \\
\hline \multirow{2}{*}{1} & \multirow{2}{*}{470} & NB & +++ & $++t$ & ++ & ++ & + & \pm & - & & \\
\hline & & MG & $+t+$ & +++ & ++ & ++ & + & - & - & & \\
\hline \multirow{2}{*}{2} & \multirow{2}{*}{210} & NB & +++ & +++ & $t++$ & ++ & + & + & \pm & - & \\
\hline & & MG & $++t$ & +++ & +++ & ++ & + & \pm & - & - & \\
\hline \multirow{2}{*}{3} & \multirow{2}{*}{223} & NB & +++ & $+t+$ & +++ & ++ & + & + & \pm & & \\
\hline & & MG & +++ & +++ & +++ & ++ & + & + & \pm & & \\
\hline \multirow{2}{*}{4} & \multirow{2}{*}{280} & NB & +++ & $+t+$ & ++ & ++ & + & + & \pm & & \\
\hline & & MG & $++t$ & $++t$ & +++ & $+t$ & + & \pm & - & & \\
\hline \multirow{2}{*}{5} & \multirow{2}{*}{190} & NB & $++t$ & $++t$ & & & & & & + & \pm \\
\hline & & MG & $++t$ & +++ & & & & & & - & - \\
\hline
\end{tabular}

Tab'e 10. The postmortem peroxidase reaction of human leukocytes. NAS-B=peroxidase staining with NAS-Benzidine solution. sec.=seconds.

\begin{tabular}{|c|c|c|c|c|}
\hline $\begin{array}{l}\text { case } \\
\text { No. }\end{array}$ & $\begin{array}{l}\text { age } \\
\text { (year) }\end{array}$ & $\begin{array}{c}\text { number of } \\
\text { hours after } \\
\text { death }\end{array}$ & $\begin{array}{l}\text { leukocytes in the } \\
\text { heart of the } \\
\text { corpses }\end{array}$ & $\begin{array}{l}\text { leukocytes on the } \\
\text { conjunctiva of the } \\
\text { corpses }\end{array}$ \\
\hline 1 & $\stackrel{\circ}{26}$ & 29 & NAS.B. 15 sec. $(+)$ & NAS.B. 20 sec. $(+)$ \\
\hline 2 & $\begin{array}{c}+9 \\
17\end{array}$ & 26 & NAS-B. 20 sec. $(+)$ & NAS.B. 20 sec. $(+)$ \\
\hline 3 & $\begin{array}{l}P \\
22\end{array}$ & 12 & $\begin{array}{r}\text { NAS-B. } 20 \text { sec. }(-) \\
60 \mathrm{sec}(+)\end{array}$ & 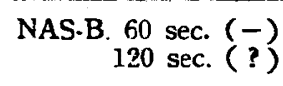 \\
\hline 4 & $\begin{array}{c}\hat{\delta} \\
19\end{array}$ & 12 & NAS-B. 45 sec. $(+)$ & $\begin{array}{r}\text { NAS-B. } 60 \text { sec. }(-) \\
120 \text { sec. }(+)\end{array}$ \\
\hline \multicolumn{3}{|c|}{$\begin{array}{c}\text { control } \\
\text { (healthy man) }\end{array}$} & $\underset{15-60 \text { sec. }(t)}{\text { NAS-B. }}$ & $\begin{array}{l}\text { NAS-B. } \\
\quad 15-120 \text { sec. }(+)\end{array}$ \\
\hline
\end{tabular}


1) In both rabbits and guinea pigs which were killed by ether inhalation, the peroxidase reacting granules of pseudoeosinophils and eosinophils, become sparse and fewer in number, abnormal in tint (turning into green or brown from blue) with increasing time lapse after death. The granules barely stain with the peroxidase stain (NAS-B solution), resulting eventually in entirely negative cells or degenerative ones. The peroxidase reactions still occur distinctly within twenty-four hours after death, while the reactions generally become negative two days after death. No. 5 in table 8 and 9 , are the animals in which the hearts were not extirpated from the thoracic cavity, and hence were not exposed to the air for two days. No. 1, 2,3 and 4 are all animals where the hearts were aseptically extirpated immediately after death and blood was aseptically withdrawn by means of the syringes to make smear preparations. As shown in table 8 and 9, when the heart is not exposed to the air, the peroxidase reaction of contained blood cells still occurs after forty-eight hours in the rabbit or sixty hours in the guinea pig. The peroxidase reactions of the blood cells remain longer when the heart is not exposed to the air than when exposed.

2) Generally speaking, as shown in these tables, the findings of peroxidase staining run parallel with those of May-Giemsa's staining in the experiments on the postmortem changes, however, in guinea pigs, the peroxidase reacting granules of the leukocytes remain longer than the May-Giemsa's granules, for example in cases of No. 1, 2, 4, 5 in table 9 the peroxidase staining granules still distinctly occurred although the May-Giemsa's staining granules never occurred in the same stage after death. This is probably due to the fact that the peroxidase reaction of guinea pig's pseudoeosinophils is generally more intense than that of rabbit's $\mathrm{s}^{28}$.

3) These postmortem changes in the peroxidase stainings were accelerated by high temperature as well as by bacterial invasions from the air or from the viscera. On the other hand, according to Tagami (1929), ${ }^{36,37}$ in the experiments with the carcases of rabbits, the phagocytic activities still remained in the leukocytes within thirty-six hours after death, and their motile activities barely disappeared in three days. Furthermore, Sugiyama (1928) ${ }^{32}$ stated that the human leukocytes in the corpse still possessed motile activities even twenty hours after death.

4) In my experiments with the leukocytes in the humanco rpses, the remarkable decrease in their peroxidase reaction could not be 
recognized. Even twenty-nine hours after death, the leukocytes in the heart of the corpses stained well with the NAS-B solution for fifteen seconds, and those on the conjunctiva also stained for twenty seconds - these staining times in the NAS-B reaction, such as fifteen or twenty seconds, do not differ from the normal (see the control in table 10). The leukocytes on the mucosa may occasionally show very weak peroxidase reactions due to the influence of secretions such as saliva, nasal or lacrimal secretions ${ }^{42}$. Again, it should be noted here that the causes of death of the corpses in table 10 were mostly intoxications by hypnotic agents. The possibility arose that these medicaments may activate the peroxidase reaction of the leukocytes in the circulating blood. In the experiments with these medicaments including phosphor, veronal, luminal, adalin, adorm (barbital + calcium), calmotin (bromural), ether etc, no remarkable influence upon the peroxidase reaction of the human leukocytes was found, although the reactions were examined after these medicaments had been added to the blood smear, not to the blood cells in the circulating blood. In short, as far as the leukocytes in the human corpses are concerned, within twenty-nine hours after death the peroxidase reacting granules still stain as distinctly as in the living state of the persons.

\section{The peroxidase staining of old blood smears.}

In examining the peroxidase reaction of the blood cells, the fresh blood smears are always desirable, because the reaction may become weaker or entirely negative with the lapse of time. However, it was made clear by the experiments with four persons that the peroxidase reacting granules in the human leukocytes are stable for as long as three years, although the peroxidase reaction becomes weaker.

After the human blood smears had been preserved in the dark room for a long time, they were stained with NAS-B solution without previous fixing. The peroxidase reaction of the leukocytes gradually became weaker with the lapse of time after the blood smear was prepared, and could not recover the same intensity as in the fresh smear. But, at least within a week, no remarkable change could be recognized. . In the eighth month after the preparation of the smear, the peroxidase reacting granules in the neutrophils became fewer as compared with those of the fresh smears, furthermore the eosinophil granules did not stain blue but brown with the NAS-B solution after one minute. However, it is interest to find that the granules of the 
leukocytes never became peroxidase negative even three years (one thousand one hundred and five days) after the preparation of the blood smears, and that, in these old smears the neutrophil granules never stained after one minute, while after two minutes with the NAS-B solution the granules were blue and coarse. On the other hand, the eosinophil granules in these old smears stained brown or green after one minute, but blackish violet after two minutes with the latter solution, furthermore, the eosinophil granules stained red very distinctly with the May-Giemsa's stain.

Suzuki and Teramoto $(1946)^{34,35}$ previously stated that the peroxidase reaction of the human leukocytes becomes more intense from the fourth week after the preparation of the smears, than in the fresh smears. A phenomenon like this was not found in this experiment, although it was distinctly confirmed in routine work that the older the smear, the more intensely the nuclei take a counterstain such as fuchsin.

Judging from all these results it can be said that the peroxidase reacting granules of the leukocytes rapidly disappear within two days under wet condition such as in the corpse, while they are preserved for as long as three years under dry condition such as air-dried smear preparation.

\section{Water solubility of the peroxidase reacting granules.}

In order to observe the influence of water on the peroxidase reacting granules in the leukocytes, and also to compare the stability of the latter granules with that of May-Giemsa's granules, two different experiments were planned using the NAS-B solution on four cases. In one experiment, the distilled water was added to the human blood smear which had already been stained with the NAS-B or MayGiemsa's stain, in another experiment, the distilled water was at first added to the blood smear before the latter staining. The $\mathrm{pH}$ value of the distilled water used was about 6.6.

Experiment 1. The human blood smears were stained with the NAS-B solution for one minute without prior fixation. Next the latter smears were immersed in the distilled water for five minutes to eight hours. It was made clear from this experiment that the blue stained granules in the leukocytes changed into the so-called double phase or entirely brown in about three or four hours after the smears were immersed in the distilled water, and that the blue granules of the 
eosinophils changed into brown more rapidly than those of the neutrophils, however, the peroxidase reacting granules in both the neutrophils and eosinophils never disappeared even at the end of eight hours. On the contrary, it was made clear by further experiment that the granules of the neutrophils stained with the May-Giemsa's stain, disappeared in one hour after the smear was immersed in the water. This suggests that the May-Giemsa's granules are more labile to the water than the peroxidase reacting ones in the neutrophil leukocytes.

Experiment 2. The fresh blood smears were at first immersed in the distilled water for five minutes to five hours, next stained with the NAS-B solution for one minute or with the May-Giemsa's stain. According to the results of the experiments, the stainable substance with the May-Giemsa's stain in the neutrophil leukocytes is more water-soluble than the stainable substance with the peroxidase stain. The stainable substance with the May-Giemsa's stain is rapidly dissolved in the water within five minutes, while that with the NAS-B solution was not dissolved at the end of five hours (table 11). On the other hand, both peroxidase and May-Giemsa's stainable granules in the eosinophil leukocytes are generally less soluble in the water than are those in the neutrophils.

The following results regarding the granules in the human leukocytes were evolved:

Table 11. The water solubility of the stainable substance with the peroxidase stain or May-Giemsa's one in the human leukocytes (Experiment 2). NB=NAS-B staining, $\mathrm{mg}=$ May-Giemsa's staining, $\mathrm{N}=$ neutrophils, $\mathrm{E}=$ eosinophils.

\begin{tabular}{|c|c|c|c|c|c|c|c|}
\hline \multirow{2}{*}{$\begin{array}{c}\text { case } \\
\text { No. }\end{array}$} & \multirow{2}{*}{$\begin{array}{c}\text { stain- } \\
\text { ing }\end{array}$} & \multirow{2}{*}{$\begin{array}{l}\text { leuko- } \\
\text { cyte }\end{array}$} & \multirow{2}{*}{$\begin{array}{l}\text { normal } \\
\text { finding }\end{array}$} & \multicolumn{4}{|c|}{$\begin{array}{l}\text { time lapse after the smear was immersed in the } \\
\text { water before the staining }\end{array}$} \\
\hline & & & & 5 minutes & 30 minutes & 1 hour & 5 hours \\
\hline \multirow{4}{*}{ I } & \multirow{2}{*}{ NB } & $\mathrm{N}$ & blue & blue & blue & blue & blue \\
\hline & & $\mathbf{E}$ & blue & blue & blue & double phase & double phese \\
\hline & \multirow{2}{*}{$\mathrm{mg}$} & $\mathrm{N}$ & neutrophil & none & none & none & none \\
\hline & & $\mathbf{E}$ & red & red & red & red & red \\
\hline \multirow{4}{*}{ II } & \multirow{2}{*}{ NB } & $\mathrm{N}$ & blue & blue & blue & blue & blue \\
\hline & & $\mathrm{E}$ & blue & blue & blue & double phase & double phase \\
\hline & \multirow{2}{*}{$\mathrm{mg}$} & $\mathrm{N}$ & neutrophil & none & none & none & none \\
\hline & & $\mathrm{E}$ & red & red & red & red & red \\
\hline
\end{tabular}


1) The stainable substance with the May-Giemsa's stain is more labile to the water than the stainable substance with the peroxidase stain.

2) The neutrophil granules stained with the peroxidase stain are more water resistant than those stained with the May-Giemsa's stain. It may be said that the peroxidase stain is more intensely adsorbed to the granules than the May-Giemsa's stain.

3) The eosinophil granules are more water resistant than the granules in the other myeloid leukocytes.

4) The eosinophil granules slowly stain blue with the NAS-B solution and rapidly become brown after water exposure. On the other hand, the neutrophil granules stain blue rapidly, and hardly change color after water immersion.

\section{The peroxidase reaction of the blood platelets.}

When stained with the May-Giemsa's or Wright's stain, azure granules of the platelets are seen in a hyaline, light blue cytoplasm, and these granules also stain with neutral red. A few mitochondrial rods and granules stain with Janus green. As to the origin of the platelets considerable theories have been proposed, but it is not yet completely determined. The platelets have been thought to be derived from the plasma itself, from erythrocytes or leukocytes, or from megakaryocyte in the bone marrow (Wright's theory). But of these, Wright's theory of megakaryocyte origin is most generally accepted to-day. Therefore the platelets may represent myelogenous cells or cell fragments. Again, the oxidase reaction of both the platelets and the megakaryocytes is regarded as positive as the myeloid leukocytes by some authors. Katsunuma ${ }^{39}$ stated that the platelets are rarely oxidase positive, whereas Tokue ${ }^{39}$ stated that their oxidase as well as peroxidase reactions are always negative. The latter investigator's viewpoint was confirmed by these experiments. The platelets both in the circulating blood and the bone marrow, did not show peroxidase positive granules using NAS-B solution. It is my opinion that the azure granules in lymphocytes and platelets are peroxidase negative by the usual staining methods.

\section{Summary and Conclusion}

The materials used in the experiments were the blood cells of healthy persons, animals (rabbits and guinea pigs) and human corpses, 
and the NAS-Benzidine (NAS-B) solution was chiefly adopted for the peroxidase stain due to its simplicity and sensitivity. The latter solution, however, does not differ essentially from the acid peroxidase stains heretofore proposed. This experimental study dealt with various inhibitors of the peroxidase reaction, postmortem changes of the blood cells, obtaining the following results :

1) The light rays exert inhibiting influences upon the peroxidase reaction of the leukocytes in the following order: Ultraviolet, visible and infra-red rays. Therefore it can be said that the longer the wave length, the weaker becomes the inhibiting influence. However, this inhibiting influence is by no means found in the erythrocytes.

2) Methanol exerts a stronger inhibiting influence upon the peroxidase reaction of the leukocytes than ethanol, however, the reaction of the erythrocytes is by no means inhibited by these alcohol, but rather is activated.

3) Excessive hydrogen peroxide solution contained in the peroxidase stain inhibits the peroxidase reaction of the leukocytes, but not that of the erythrocytes.

4) The erythrocytes are peroxidase negative under neutral or alkaline condition, while the myeloid leukocytes are positive under both acid and alkaline conditions.

5) The peroxidase reaction positive granules in the leukocytes show various colors-yellow, brown, green, blue, purple, black and other transitional colors. This is, I infer, due to the changes of the benzidine derivatives ${ }^{19,43}$ caused by various factors such as heating, fixation and $\mathrm{pH}$ etc.

6) Generally speaking, the erythrocytes possess stable peroxidative activities, while the leukocytes possess labile peroxidative activities. The peroxidase reacting substance of the erythrocytes is light raysstable, heat-stable, $\mathrm{H}_{2} \mathrm{O}_{2}$-stable, and alcohol-stable.

7) It is interesting to note that the granules in the leukocytes which were made entirely peroxidase negative by the inhibitors such as sunlight, ultraviolet rays or alcohol, still stain very finely with the May-Giemsa's stain. This is one of the evidences that the peroxidase reacting granules differ from the May-Giemsa's or Wright's staining special granules in the leukocytes. Again, such a difference between these two granules is also found in the experiment on the postmortem changes; the peroxidase reacting granules in guinea pig's leukocytes are preserved longer than the May-Giemsa's special granules of the same leukocytes. 
8) As far as the leukocytes in the human corpses are concerned, the peroxidase reacting granules still stain as distinctly as in the living state of the subjects, within twenty-nine hours after death. In examining the postmortem changes of rabbits and guinea pigs, the peroxidase reacting granules in the leukocytes disappear within two days after death, however, these granules are preserved longer when the heart containing blood is not exposed to the air than when exposed.

9) The granules of the leukocytes on air-dried blood smear never become peroxidase negative even three years after the preparation of the smear. It is of interest that the peroxidase reacting granules disappear within two days under wet conditions such as in the corpses, whereas they are almost intact at the end of three years under dry conditions such as air-dried blood smear.

10) The stainable substance with the May-Giemsa's stain in the human neutrophil leukocytes is rapidly dissolved in the distilled water (pH 6.6) within five minutes, while the stainable substance with the peroxidase stain (NAS-B solution) resists dissolution for five hours.

11) The eosinophil granules are always more stable under the various unsuitable conditions utilized in this experiment than the neutrophil granules.

12) The blood platelets both in the circulating blood and bone marrow, are peroxidase negative.

I am indebted to Professor Mitsui for the valuable advice on this study, and also wish to express sincere thanks to Dr. Shinnosuke Saéki of the Government Chemical Industrial Research Institute, Tokyo, and to Dr. Shūichi Ohtake of Tokyo College of Science for the spectroscopic test of the infra-red filter used, further I am grateful to my younger sister Hiroko for her kind aid during this work.

This study was carried out with the grant in aid for fundamental scientific research.

\section{References}

1) Agner, K.: Acta physiol. Scand., 2, Suppl. 7, 1941.

2) - Detoxicating effect of verdoperoxidase on toxins. Nature. 159 (4034): 271-272, 1947.

3) A rakawa, T.: Quantitative determination of human milk peroxidase. 30PR. Tohoku J.E.M., 16(1, 2):97-106, 1930.

4) Bansi, H.W. and Ucko, H.: Klin. Wochenschr., S. 309, 1927.

5) Chiba, M. and Abe, J.: Short-timed peroxidase reaction of blood leukocyte. 40PR. Tohoku J.E.M. 19(5, 6):479-485, 1932. 
6) Chiba, M.: Prolongation of short-timed peroxidase reaction of leucocytes. 41PR. Tohoku J.E.M. 19(5, 6):486-512, 1932.

7) Cowdry, E.: Textbook of Histology, London, Henry Kimpton. p42-43, 1934.

8) Getchell R.W. and Walton, J.H.: J. biol. Chem., 91, p119, 1931.

9) Gradwohl, R.B.H.: Glinical laboratory methods and diagnosis. St. Louis. Vol. 1, p609-610, 1948.

10) Kamimaé, T.: Enzymology. Shibundo, Tokyo, p508-509, 1950. (Japanese)

11) Kato, K.: New Clinical Hematology. Bunkodo, Tokyo, p27-28, 1949. (Japanese)

12) Lemberg, R. and Legge, J.: Hematin compounds and bile pigments. Interscience publishers, Inc., New York, pp402, 403, 436, 1949.

13) Mitsui, T. and Ikeda, S.: NAS-Benzidine reaction of blood cells. Ist Report. Okaj. fol. anat. jap, 23(4-5) : 331-336, 1951.

14) _ - On the four phases of peroxidase staining of blood cells. 2nd Report. Okaj. fol. anat. jap. 23(4-5):323 -329, 1951.

15) __ Morita, Sasaki and Tsuka moto: Comparison among various peroxidase staining methods. 7th Report. Okaj. fol. anat. Jap. 24(2): 99-101, 1952.

16) -Sasaki, Tsuka moto and Morita: On the difference between peroxidase reacting substances in the leukocytes and erythrocytes. I. Medicine and Biology. 24(3): 87-89, 1952. (Japanese)

17) __ , - II. Medicine and Biology. 24(6): 215218, 1952. (Japanese)

18) Morita, F,: On the three phases of peroxidase reaction of blood cells and the limit of benzidine and hydrogen peroxide. Shika Geppo. 24(6-12):15-16, 1951. (Japanese)

19) : Effect of streptomycin upon the peroxidase staining of blood cells. 6th Report. Okaj. fol. anat. jap. 24(2): 95-98, 1952.

20) Nihon Gakujitsu Kaigi: Clinical report on atomic bomb. Tokyo. p61-62, 1952. (Japanese)

21) Nord, F.: Advances in Enzymology. Vol. 7. Interscience publishers, Inc., New York, p266-267, 1947.

22) Ohtani, T.: Jikken Koso Kagaku. Kagakushoin, Tokyo, p5, 1939. (Japanese)

23) Oppenheimer, C.: Die Fermente und ihre Wirkung. Bd. II, S. 1633-1643, 1939.

24) Osgood, E.E.: Atlas of Hematology. San Francisco, Stacey \& Co., 1938.

25) Sasaki, M.: Influence of fixatives upon the peroxidase staining. 8th Report. Okaj. fol. anat. jap. 24(3): 193-197, 1952.

26) Sato, A. and Sekiya, S.: New peroxidase reaction (copper method). Tohoku Igaku Zasshi. Vol. 6, p534-541, 1922. (Japanese)

27) _ and A A simple method for differentiation of myeloic and lymphatic leucocytes of the human blood. 3PR. Tohoku J.E.M. 7(2):111-115, 1926.

28) - and Suzuki, T.: Normal blood leucocytes of domestic animals, as they appear under the copper peroxidase reaction. 90PR. Tohoku J.E.M. 32(1,2): 91-96, 1938.

29) Sato, S.: Studies on time lapse after death. Nihon Hōigaku Zasthi. 5(5):1-13, 1951. (Japanese)

30) Sa to, T. and Abe, Y.: Study on the peroxidase reaction of blood cells. Act. hem. jap. 14(4): 72, 1951. (Japanese)

31) Shōji, K.: The copper peroxidase reaction of animal blood. 10PR. Tohoku J.E.M. 10(4) : 293-298, 1928.

32) Sugiyama, S.: New studies and methods on the blood and tissue. Nankodo, 
Experimental Study on the Peroxidase Reacting Granules in the Blood Cells.

Tokyo, 3 ed., pp23-24, 38, 53, 1952. (Japanese)

33) Suzuki, K. and Arakawa, T.: Aperoxidatic milk as a possibly early symptom of avitaminosis-B. 34PR. Tohoku J.E.M. 16(3-4): 228-231, 1930.

34) Suzuki, T.: Copper method, temperature and old blood smears. Act. hem. jap. $8(1): 8,1944$. (Japanese)

35) Suzuki, T. and Teramoto, Y.: On the temperature and old blood smears in the peroxidase reaction. Act. hem. jap. 9(1):9-12, 1946. (Japanese)

36) Tagami, K.: Changes of the blood cells in corpses. Juzenkai Zasshi. Vol. 34, p1653, 1929. (Japanese)

37) —- : Shapes of the nuclei of the leucocytes in the human corpses. Juzenkai Zasshi, Vol. 37, p182, 1932. (Japanese)

38) Takada, T.: Essential Physics. Dainihon Insatsu. Tokyo, p361, 1949. (Japanese)

39) Tokué, K.: The oxidase and the peroxidase reaction in different leukocytes of the blood. 25PR. Tohoku J.E.M. 12(5, 6): 445-458, 1929.

40) Tsukamoto, K.: Study on the influence of temperature upon the peroxidase reaction of blood cells. 5th Report. Okaj. fol. anat. jap. 24(2):89-94, 1952.

41) - Influence of temperature upon the peroxidase reaction of blood cells. Medicine and Biology. 22(2): 68-70, 1952. (Japanese)

42) - Sasaki and Morita: NAS-Benzidine reaction of the salivary corpuscles. The report of the scientific council of the Japan dental association in 1951. p141, 1952. (Japanese)

43) __ - Electron micrographs of blood cells with special reference to the crystals produced by the peroxidase reaction. 9th Report. Okaj. fol. anat: jap. 24(3): 199-203, 1952.

44) Tsujimura, S.: Study on the peroxidase reaction of blood cells. Act. hem. jap. 13(6), 14(1, 4, 5), 1950-1951. (Japanese)

45) Usami, K. and Yamamoto, Y.: New peroxidase reaction using zinc sulfate. Act. hem. jap. 8(3): 181-183, 1943. (Japanese)

46) Wintrobe, M.M.: Clinical. Hematology. 2 ed., Lea \& Febiger, pp158, 187-190, 1949. 